\title{
MASCULINO DE 81 ANOS CON MUCOCELE APENDICULAR \\ Cistoadenoma tubulovelloso de Apéndice.
}

\author{
Raisa Zuwolinsky Elguera ${ }^{1}$ \\ Carlos Barrantes León ${ }^{2}$
}

\footnotetext{
${ }^{1}$ Medico General. Universidad de Costa Rica. Correo electrónico: raizuw@gmail.com

${ }^{2}$ Especialista en Cirugía General. Asistente del servicio de Cirugía General Hospital Max Peralta Jiménez. Correo electrónico: barranleon@hotmail.com
}

\section{RESUMEN}

Se presenta caso de paciente masculino de 81 años, vecino de Cartago con antecedentes patológicos personales de colelitiasis e hipertensión prostática benigna, quien es referido al hospital Max Peralta Jiménez en marzo del 2014 por historia de dolor abdominal de larga data y con ultrasonido previo que determina la presencia de masa abdominal en fosa iliaca derecha. Es visto por el servicio de cirugía general del mismo hospital en donde se completan estudios diagnósticos y posteriormente es llevado a sala de operaciones con diagnóstico de mucocele apendicular, se realiza laparotomía exploratoria y colectomía derecha con ileo-transverso anastomosis. Los estudios histopatológicos posteriores confirman el diagnóstico de mucocele apendicular asociado a adenoma túbulo velloso muco-productor.

\section{PALABRAS CLAVE}

Adenoma. Mucocele. Apendicectomia. Hemicolectomia derecha.

\begin{abstract}
We presented a case of an 81 year old male patient with a personal history of cholelithiasis and benign prostatic hyperplasia, who is referred to the hospital with a history of abdominal pain associated to an abdominal mass on the right iliac fossa, discovered on a previous abdominal ultrasound. In the hospital, diagnostic studies are completed, he is then taken to the operation room
\end{abstract}

with a diagnosis of appendiceal mucocele, exploratory laparotomy and right colectomy was performed with ileo-transverse anastomosis. Histopathological studies further confirmed the diagnosis of appendiceal mucocele associated with mucinous tubulovillous adenoma.

\section{KEY WORDS}

Adenoma. Mucocele. Appendectomy. Right hemicolectomy.

\section{CASO CLÍNICO}

Paciente masculino de 81 años, vecino de Cartago, tabaquista, con antecedentes patológicos personales de colelitiasis e hipertensión prostática benigna, quien es referido del primer nivel de atención el 13 de marzo del 2014 con historia de dolor abdominal de larga data, distención abdominal, astenia y adinamia, al cual se le realizo ultrasonido de abdomen en marzo del mismo año con evidencia de masa solida abdominal, de $128 \times 75 \times 112 \mathrm{~mm}$ en fosa iliaca derecha, de aspecto heterogéneo, bordes definidos y flujo vascular detectable, con aumento de la ecogenicidad de la grasa mesentérica, sin evidencia de líquido o colecciones.

Es visto en consulta externa del servicio de cirugía general del hospital Max Peralta Jiménez, en donde se indican estudios diagnósticos. Se realiza colonoscopia, la cual reporta compresión extrínseca a nivel de ciego como único hallazgo patológico; además de marcadores tumorales 
con valor de $13 \mathrm{mcg} / \mathrm{L}$ para el antígeno carcinoembrionario; posteriormente se realiza tomografía axial computarizada con medio de contraste intravenoso, la cual evidencia una lesión quística de contenido grumoso localizada en fosa iliaca derecha, ovalada de 122 × 75 × 80 $\mathrm{mm}$ con mínimo realce homogéneo, la cual desplaza asas intestinales, principalmente la pared medial del ciego, sin evidencia de adenopatías peri lesionales.

En junio del mismo año, con el objetivo de realizar una intervención quirúrgica, es ingresado al servicio de cirugía general del hospital Max Peralta Jimenez, a la exploración física se documenta una masa sólida, indurada, móvil, de aproximadamente $5 \mathrm{~cm}$ de diámetro, no dolorosa a la palpación. Es llevado a sala de operaciones con diagnóstico pre y post quirúrgico de mucocele apendicular, se realiza laparotomía exploratoria con evidencia de masa quística de contenido mucoso de aproximadamente $30 \mathrm{~cm}$ adherida a ileon distal, que impresiona ser de origen apendicular, la cual se perfora durante la manipulación, se lleva a cabo colectomía derecha e ileo-transverso anastomosis sin complicaciones. La pieza quirúrgica es llevada al servicio de patología del mismo hospital en donde los estudios histopatológicos determinan el diagnóstico de mucocele apendicular, adenoma túbulo velloso apendicular mucoproductor con atipia moderada.

El paciente evoluciona sin complicaciones hasta el egreso y posteriormente continua su seguimiento en el servicio de cirugía general.

Figura 1. Mucocele Apendicular.

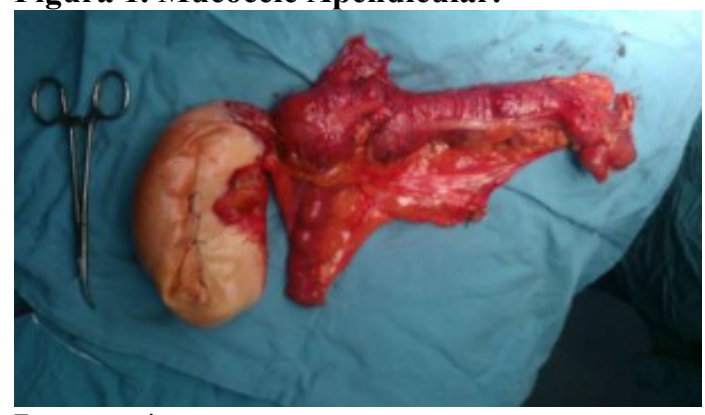

Fuente propia

\section{DISCUSIÓN Y ANÁLISIS DEL CASO}

Los tumores del apéndice cecal se encuentran presentes en menos del $1 \%$ de los especímenes de apendicectomias. ${ }^{(1)}$ Se estima que su incidencia es de 0,12 casos por cada 100 mil individuos por año. ${ }^{(1)}$ Según sus características histológicas se clasifican en neoplasias epiteliales y no epiteliales. ${ }^{(1)}$

Los tumores epiteliales corresponden al grupo más frecuentemente reportado, varias clasificaciones han sido propuestas con el objetivo de categorizar estas lesiones. ${ }^{(1-5)}$ En el año 2005, Pai y Longacre proponen clasificar dichos tumores en adenomas mucinosos, tumor mucinoso de potencial maligno indeterminado, tumor mucinoso de bajo potencial maligno y adenocarcinomas. Por otro lado, la organización mundial de la salud los ha clasificado en adenoma, tumores mucinosos de bajo grado $\mathrm{y}$ adenocarcinoma. $^{(1-5)}$

Los adenomas apendiculares se definen como lesiones benignas de la mucosa glandular, sin invasión estromal, que una vez que son resecados por completo no presentan riesgo de recurrencia. ${ }^{(1,2,4,5)}$ Poseen el mismo patrón de crecimiento que los adenomas en el resto del intestino grueso, clasificándose según este en tubulares, vellosos, túbulo-vellosos y serrados. ${ }^{(2)}$ El adenoma túbulo-velloso es el más infrecuente, se ha identificado únicamente en $0,06 \%$ de las piezas quirúrgicas post apendicectomía. ${ }^{(6)}$

Los adenomas mucinosos apendiculares se caracterizan por presentar atipia leve o moderada, mitosis aumentadas, proliferación de células epiteliales mucinosas y en algunas ocasiones perforación. ${ }^{(1,3,5)}$ La acumulación de mucina en el apéndice secundaria a estas lesiones se conoce como cistoadenoma, término que en este contexto se utiliza de manera intercambiable con adenoma .. $(2,4,5)$ Sin embargo, la descripción macroscópica de la dilatación apendicular como consecuencia de la acumulación de moco en el lumen se conoce como mucocele apendicular. $^{(2,7,8)}$ Esta condición está presente en $0,3 \%$ de las apéndices quirúrgicas, y entre el 63 y $84 \%$ de los casos la causa corresponde a cistoadenomas. $^{(7,8,9)}$. No obstante, pueden ser ocasionados por otras causas tanto neoplásicas como inflamatorias. ${ }^{(7)}$

Los cistoadenomas del apéndice cecal se presentan en todas las edades, sin embargo se encuentran más frecuentemente en pacientes entre los 45 y 65 años. ${ }^{(6)}$ En el caso de los mucoceles, estos se presentan principalmente en pacientes del sexo femenino, con una edad media 
de 50 años. $^{(9)}$ Puede permanecer como un proceso patológico asintomático o presentarse con dolor crónico en el cuadrante inferior derecho abdominal, una masa pélvica, hernias de la pared abdominal con contenido de mucina o carcinomatosis peritoneal con o sin ascitis; sin embargo su hallazgo es principalmente incidental durante la realización de estudios de imágenes o posterior a apendicectomía por apendicitis..$^{(1,4,5,7,8)}$ En algunas ocasiones se han reportado síntomas inespecíficos como distención abdominal, aumento del diámetro abdominal, ganancia de peso, fatiga, disnea, saciedad temprana, diarrea o melena. ${ }^{(1,4,6,8)}$

El diagnostico pre quirúrgico se logra en escasas ocasiones. Se deben llevar a cabo estudios de imágenes como ultrasonido, ultrasonido endoscópico, tomografía axial computarizada y/o resonancia magnética. ${ }^{(7)}$ Típicamente son lesiones sésiles, cuya dilatación luminal puede alcanzar hasta 6 centímetros de diámetro. En los estudios de imágenes se puede determinar la naturaleza quística de la lesión, la presencia de calcificaciones y otras características inespecíficas. ${ }^{(7)}$ En los casos en que se asocia el mucocele, se puede observar en la colonoscopia una masa blanda con orificio central del cual fluye mucina, esto que se conoce como el signo del volcán. ${ }^{(7)}$

Se deben realizar además marcadores tumorales como CA 19-9, antígeno carcino embrionario, CA 125 , estos pueden estar elevados en $60 \%$ de los casos de malignidad, no obstante el diagnóstico definitivo se obtiene en la mayoría de los casos mediante estudios histopatológicos posteriores a la intervención quirúrgica. ${ }^{(1,4,6)}$ la pieza quirurgica debe evaluarse exhaustivamente, debido a que existe gran dificultad en diferenciar los adenomas apendiculares de otros tumores epiteliales, principalmente de neoplasias de potencial maligno indeterminado y bajo potencial maligno o tumores mucinosos de bajo grado. ${ }^{(2,4,5)}$

Es importante una vez confirmado el diagnostico realizar vigilancia mediante colonoscopia debido a que se ha documentado la existencia de lesiones epiteliales sincrónicas en el otras partes del intestino grueso así como también lesiones mucinosas de ovario. ${ }^{(2,4)}$

Los mucoceles apendiculares, y en general los adenomas mucinosos, pueden presentar complicaciones como intususcepción, obstrucción intestinal, sangrado o ruptura. ${ }^{(7,8)}$ Aproximadamente en $20 \%$ de los casos de cistoadenomas ocurre ruptura apendicular como un evento secundario al aumento de presión luminal, el contenido de moco entra a la cavidad peritoneal en donde sigue el flujo de líquido peritoneal en sentido de las manecillas del reloj. ${ }^{(1,7,8)}$ Sin embargo en los casos de adenomas productores de mucina, el contenido vertido a la cavidad peritoneal no contiene células neoplásicas por lo que no se asocia a siembras peritoneales, condición conocida como pseudomixoma peritonei y que se encuentra asociada a otros tumores epiteliales del apéndice cecal. $^{(2,8)}$

Los adenomas pueden presentar degeneración maligna dependiendo del tamaño tumoral y el tipo patológico, siendo de mayor riesgo aquellos que miden 2 centímetros o más, sin embargo las lesiones menores a $1 \mathrm{~cm}$ presentan hasta $5 \%$ riesgo de malignizar. ${ }^{(6)}$ Según su patrón de crecimiento presentan mayor riesgo los adenomas vellosos, seguido por los túbulo vellosos y luego los tubulares. ${ }^{(6)}$

Entre los diagnósticos diferenciales de estas lesiones se deben debe incluir: patología benigna de apéndice, quistes mesentéricos, hidrosalpinx, tumor carcinoide, linfomas, endometriosis. Tambien se deben descartar otras causas de mucocele apendicular como quistes de retención o mucocele simple, hiperplasia epitelial o cistoadeno carcinoma $u$ otras neoplasias mucinosas. $^{(5,7,8)}$

El tratamiento de elección de las neoplasias epiteliales del apéndice es siempre quirúrgico, sin embargo la extensión de la resección depende de las características de la lesión. En general es aceptado llevar a cabo apendicectomía con margen de resección libre de enfermedad para los casos de adenomas apendiculares, se asocien estos o no a mucoceles. ${ }^{(7)}$ Sin embargo, algunos autores recomiendan que en masas mayores de 2 centímetros se lleve a cabo una hemicolectomia derecha. ${ }^{(9)}$ En los casos de neoplasias de potencial maligno indeterminado o neoplasias de bajo grado en los que no se logra obtener márgenes de resección libre de enfermedad, se recomienda realizar resección de ciego. ${ }^{(2,4,6,7)} \mathrm{En}$ lesiones malignas el tratamiento de elección es hemicolectomia derecha; sin embargo algunos autores recomiendan realizar apendicectomía en 
los casos en los que es posible obtener márgenes libres y no se encuentra evidencia de invasión nodal. ${ }^{(4,7,9)}$

La manipulación de la masa tumoral es de especial cuidado durante la cirugía, se debe evitar la ruptura de la lesión para disminuir el riesgo de derramar su contenido en la cavidad abdominal. ${ }^{(7,8)}$ Siempre que se encuentre material mucinoso en la cavidad abdominal se debe llevar a cabo un estudio citológico para descartar la presencia de células epiteliales, esto cobra especial importancia en aquellos casos en los que no es posible realizar el diagnóstico definitivo previo al tratamiento. ${ }^{(7,8)}$

El pronóstico para estas lesiones benignas es favorable, se ha reportado una sobrevida a los 10 años de $91 \%$. $^{(7)}$ En general se recomienda seguimiento de los pacientes posterior al tratamiento a pesar de la naturaleza benigna de estos tumores, sin embargo no existe un régimen específico para estas lesiones por lo que en algunos casos se recomienda el mismo seguimiento que en lesiones malignas, siendo este cada 6 meses durante los siguientes dos años y posteriormente anual hasta cumplir 5-10 años de la resección. ${ }^{(7)}$

\section{CONCLUSIONES}

Los tumores epiteliales de apéndice son neoplasias que a pesar de ser poco comunes representan el grupo más importante de tumores apendiculares. Conocer su clasificación, diagnóstico y manejo es de vital importancia para el médico tratante.

Los mucoceles apendiculares pueden resultar de adenomas $\mathrm{u}$ otros tumores epiteliales. El diagnóstico pre quirúrgico de estas lesiones se obtiene en pocas ocasiones y su tratamiento siempre es quirúrgico, por lo se debe tener especial cuidado en la manipulación de las lesiones y toma de muestras para citología de la cavidad abdominal, además de valorar cada caso de manera individual con el fin de determinar el tratamiento adecuado según las características tanto del paciente como de la lesión.

\section{BIBLIOGRAFÍA}

1. Shankar $\mathrm{S}$ Ledakis $\mathrm{P}$ Halabi $\mathrm{H}$ Gushchin V Sardi A Neoplasms of the Appendix, Current Treatment Guidelines. Hematol Oncol Clin N Am 2012; 26: 1261-1290.

2. Doyle V. Bateman A. Pathology of appendiceal tumours. Surgery 2007; 25(9): 363-367.

3. Misdraji J. Appendiceal Mucinous Neoplasms. Arch Pathol Lab Med 2010; 134: 864-871.

4. Pai R. Longacre T. Appendiceal Mucinous Tumors and Pseudomyxoma Peritonei Histologic Features, Diagnostic Problems, and Proposed Classification. Adv Anat Pathol 2005; 12(6): 291-311.

5. Robert D. Odze and John R. Goldblum. Surgical Pathology of the GI Tract, Liver, Biliary Tract and Pancreas. Elsevier 3 edición, 2015.

6. Bodin R. Peycru T. Schwartz A. Jarry J. Pommier N. Durand-Dastes F. Tubulovillous adenoma of the appendix: $A$ case report and review of the literatura. Gastroentérologie Clinique et Biologique 2010; 34: 633-635.

7. Caracappa D. et al. Appendiceal mucocele, a case report and literatura review. Ann Ital Chir 2011; 82: 239245.

8. Dhage-Ivatury S. Sugarbaker P. Update on the Surgical Approach to Mucocele of the Appendix. J Am Coll Surg. 2006; 202(4): 680-684.

9. Sierra E. Sierra G. Leone G. Quiñones C. Salazar V. Cistoadenoma mucinoso de apéndice. Informe de un caso. Cir Cir 2010; 78: 257-260.

\section{CONFLICTO DE INTERES}

Los autores declaran que no existe ningún conflicto de interés en el presente reporte. 